\title{
A Study on Fungal Etiological Agents in Suppurative Keratitis
}

\author{
Anna Cherian*, Honey Susan Paul, R. Jyothi and J.T. Ramani Bai \\ Department of Microbiology, Government Medical College, Trivandrum, Kerala, India \\ *Corresponding author
}

\section{A B S T R A C T}

Fungal keratitis is a major ophthalmic problem. In view of the importance in timely diagnosis and treatment of keratitis which would otherwise lead to serious complications like loss of vision, hypopyon formation, endopthalamitis, etc., this study was intended to bring into light the fungal etiological agents in suppurative keratitis. The specimen was

Keywords

Suppurative

keratitis,

Corneal scrapings, SDA.

Article Info

Accepted:

23 May 2017

Available Online:

10 June 2017 collected from suppurative keratitis cases irrespective of prior antibiotic treatment during the period of one year from March 2012 to February 2013. Those cases with loss of corneal epithelium with signs of inflammation, and stromal infiltration were taken as suppurative keratitis cases. Five or more corneal scrapings are to be taken, based on the condition of the patient. The corneal scrapings are used for $10 \% \mathrm{KOH}$ mount and Gram staining. Sabouraud's Dextrose Agar (SDA) is inoculated by touching the medium with the needle. Extreme care is taken to keep the entire process sterile. During the one-year period, out of the 100 patients with suppurative keratitis, there were $56(82.36 \%)$ fungal isolates. Aspergillus spp. was the commonest, constituting $22(39.29 \%)$ of the total fungal isolates. Fusarium is the second most common in this study $9(16.7 \%)$. Although corneal ulceration is a very rare cause of mortality, it is one of the important causes of morbidity and disability in South Kerala, especially among lower socio-economic groups. The present study gives an insight about the fungal aetiological agents of suppurative keratitis in this part of Kerala. The results of this study are similar to that in other parts of India. These findings have important public health implications for the treatment and prevention of corneal ulceration in the developing world.

\section{Introduction}

Corneal ulcers pose a challenging problem to the ophthalmologists, as their etiology is of varied origin and their course is unpredictable. Prognosis is uncertain. A specific therapy is still a confused issue, as effective specific antifungal broad-spectrum agents are not still available. Corneal ulcers occur more frequently in elderly debilitated, arteriosclerotic and anaemic persons. They occur among the lower classes exposed to injury and they are prone to neglect the infection. They occur specially during harvest season and by the time the patient seeks medical aid, the ulcers are much advanced and they may recover without perforation but with opacities of varying density or finally end in perforation with blindness as its sequelae. Major causes of blindness in India are: - Cataract - 62.6\%, Refraction Error $19.7 \%$, Glaucoma - 5.8\%, corneal pathologies $0.9 \%$ and other causes- $11.0 \%$ (Arlappa). Wide geographical variation exists in the epidemiology of microbial keratitis (MK) based on economic and climate factors. 
Fungal keratitis (FK) accounts for about 50\% of all cases of culture-proven $\mathrm{MK}$ in some developing countries. The protracted course of the condition and the diversity of clinical presentation may contribute to unfavorable outcomes (Ibrahim et al., 2011).

Fungi gain access into the corneal stroma through a defect in the epithelium, then multiply and cause tissue necrosis and an inflammatory reaction. The epithelial defect usually results from trauma (e.g., contact lens wear, foreign material, prior corneal surgery). The organisms can penetrate an intact descemet membrane and gain access into the anterior chamber or the posterior segment. Mycotoxins and proteolytic enzymes augment the tissue damage (Kindo et al., 2009).

Incidental ocular trauma by plant leaves or vegetative materials is a facilitating factor in most patients, which can lead to fungal keratomycosis and corneal ulcer after the release of cytotoxins. The inflammatory reaction occurs after the release of mycotoxin and proteolytic enzymes, which are responsible for the catastrophic corneal melting in some patients (Tabatabaee et al., 2013).

A number of studies indicate that indiscriminate use of antibiotics and corticosteroids potent actual cause of the infestation by fungi. Antibiotics and corticosteroids have been variously implicated separately or in combination for this potentiation. Steroids either in combination with antibiotics or without them are freely prescribed not only by ophthalmologists but also by general practitioners and nonophthalmic specialists. The abuse of corticosteroids has done more harm than good as in herpetic keratitis and has hastened the progression of the superficial diseases of the cornea to perforation and made the opportunistic fungi like Cephalosporium,
Penicillium and Fusarium species to become pathogenic. Secondly the suppressive, antiinflammatory effect of corticosteroids may delay the clinical diagnosis of a possible fungal infection which is partly made from the antibiotic resistant characters of fungal infections (Srinivasan, 1969).

The purpose of the study is to evaluate the current pathogens causing fungal keratitis at tertiary care centre.

\section{Materials and Methods}

Specimens were collected from both OP and IP patients with suppurative keratitis in a minor theatre and inoculations done immediately. After properly cleaning eye with normal saline, a drop of local anaesthetic (proparacaine $0.5 \%$ ) was instilled, then eyes were focused on a slit lamp, and the corneal ulcer cleaned with sterile cotton swab soaked in sterile saline. Scrapings were taken from centre, edges, and base of the ulcer with a No. $26 \mathrm{G}$ needle. Five or more scrapings were taken based on the condition of the patient. The corneal scrapings taken were used for $10 \% \mathrm{KOH}$ mount. Another specimen was inoculated by making a well with a needle on Blood agar and MacConkey agar. Sabouraud's Dextrose Agar (SDA) was inoculated by touching the medium with the needle. Extreme care is taken to keep the entire process sterile. Study period was from February 2012 to March, 2013.

\section{Results and Discussion}

Out of 100 patients included in the study, 68 were males, and 32 were females. Age of patients included in the study ranged from 1082 years, and keratitis cases were maximum in 41-50 year age group (29\%), followed by 51-60 years (19\%). Minimum incidence was found to be in extremes of age. 
Occupational profile of the patients included in this study showed a maximum incidence among manual labourers, followed by housewives and students. $66 \%$ of patients included in this study were from rural areas, $22 \%$ from semi-urban areas, and 12\% from urban areas. $82 \%$ of patients included in this study belonged to the low socio-economic group. Among the 56 fungal isolates, 39 cases occurred in the rainy season (May-July). Prior treatment was taken by $63 \%$ of patients included in this study. Topical antibiotics alone were taken by $55.5 \%$ of patients, and $57 \%$ of patients gave history of ocular trauma. $25.39 \%$ of patients took topical antibiotics with topical antifungals.

Corneal blindness is a major public health problem worldwide and infectious keratitis is one of the predominant causes. Corneal infection of fungal aetiology is very common and represents $30 \%$ to $40 \%$ of all cases of culture-positive infectious keratitis in South India. Fungal keratitis is a major ophthalmic problem. In view of the importance in timely diagnosis and treatment of keratitis which would otherwise lead to serious complications like loss of vision, hypopyon formation, endopthalamitis, etc, this study was intended to bring into light fungal etiological agents. In the present study, fungal isolates were 56 $(82.36 \%)$ of the total 68 isolates.

Of the 100 patients included in the study 68 were males and 32 were females. Male: Female ratio is 2.125:1.This finding is in accordance with the results got in studies done by Punia et al., (2014); Bharati et al., (2014) and Saha et al., (2006) in India. In this study, age of patients ranged from 10-82 years, and keratitis was maximum in 41-50 year age group (29\%), followed by 51-60 years $(19 \%)$, and $31-40$ years $(15 \%)$.

Minimum incidence was found to be in extremes of age groups. In the age group with more than 70 years, it was found to be $4 \%$, and in the less than 10 years age group, it was $1 \%$. Similar results were obtained in studies done by Narayan (2013). In his study, done in Oman majority $(64.36 \%)$ of them were over 60 years of age.

Majority of the patients included in this study were manual laborers which included construction workers, factory workers, and non-specific agriculture related workers. Farmers constituted less than $10 \%$ of the cases. This may be due to the decreasing areas of agricultural land, and a dwindling agricultural workforce in South Kerala. The other significant groups were housewives (12\%), students (9\%), and office-workers (4\%). But, farmers were mostly affected in studies done in Vishakaptnam (Anuradha et al., 2013) and Bhopal (Sharma et al., 2013).

In majority of the studies, keratitis cases occurred commonly in low income groups, and a similar occurrence was noted in rural population. In this study, about $66 \%$ cases were rural population, $22 \%$ semi-urban, and $12 \%$ belonged to urban population. Similar finding was got in a study done in Bhopal. Rural residents have shown the higher incidence $(83.1 \%)$ of keratitis in north Karnataka also $82 \%$ of patients who participated in the study belonged to low socioeconomic group, $16 \%$ to middle socioeconomic group and $2 \%$ to high socioeconomic group. In the study major predisposing factor for keratitis is found to be the history of corneal injury. This could be attributed to the fact that majority of patients were rural residents, belonged to low socioeconomic group and majority were manual labourers. Hence, their working and living environment exposed them to the threat of foreign body fall and the resulting corneal ulcer. Geographical location and climate are reported to influence the clinical presentation and outcome of fungal diseases, probably 
through changes in the causative agent (Rautaraya et al., 2011). The incidence of fungal keratitis was higher in June, July and August. Maximum number of keratitis cases was seen in the months of July to October followed by November to February in north Karnataka. The peak incidence occurred in the winter months of June-August, during which there were $73(38 \%)$ admissions in Australia (Butler et al., 2005).

$63 \%$ of patients had taken treatment from outside before being referred. $37 \%$ of patients did not receive any treatment. This is a slightly higher percentage considering the fact that many of them had treatment from outside. This may be because $55.56 \%$ of patients had taken topical antibiotics alone and were not treated for fungus. Moreover, $25.4 \%$ of patients were on a combination treatment with topical antibiotics and antifungals, pointing to the awareness among the general practitioners about the increasing prevalence of fungal corneal ulcers. This demonstrates a very welcome trend, along with the fact that only 4 of the 100 cases were previously put on medications containing steroids. On the other hand, this may be due to the increasing availability of ophthalmologists in rural areas, and the increasing awareness regarding the seriousness of the corneal injury among the public. This can also be supported by the fact that majority of patients visited a medical practitioner in 2-3 days of occurrence of injury. Medical treatment was documented in 640 (93.2\%) of the 687 patients before coming to the National Ophthalmology hospital in North Vietnam.

In this study, $57 \%$ gave history of ocular trauma prior to corneal ulceration, while $43 \%$ did not give any obvious history of injury. Corneal injury is the most important predisposing cause (and often the precipitating cause) of corneal ulceration, especially in the developing countries where most of the population is daily wage manual labourers. The results obtained in this study are consistent with results obtained from different parts of India (Kumari et al., 2002). Corneal injury was caused by substances like sand dust, which contributed to $29 \%$. Other particles which caused injury were wooden piece, building material, vegetative matter, insects, fingernail, and dirty water. None of the injuries reported in this study were caused by paddy husk which was one of the major causes in Central Kerala in the past, when rice cultivation was common.

In developed countries where agriculture and labor are largely mechanized, other predisposing factors become more important.

However, trauma still remains as one of the major predisposing factors in the development of corneal ulcers in certain developed countries like Franc, Switzerland, Australia, and USA (Schaefer et al., 2001; Gebauer et al., 1996). According to Elisabeth Karsten, et al., trauma is quite commonly associated with infection by filamentous fungi such as Fusarium solani, Aspergillus spp and Bipolaris spp; especially when the trauma is caused by vegetative matter particularly in tropical climates.

In a retrospective study done on Fusarium keratitis in Taiwan, major predisposing factors were found to be corneal trauma with vegetable matter, or organic matter, usage of contact lens, and steroid use. Contact lens usage, although the commonest predisposing factor in developed countries (Bozorgmehr et al., 2011) was conspicuously absent in this study. This can be attributed to the virtual absence of contact lens usage among the low income group. It is also prudent to think that the contact lens users would prefer private hospitals for treatment. 
Eight patients in the study were suffering from diabetes mellitus. Similar finding was obtained in a study done in Gujarat (Hoffart et al., 2012).

Out of the 56 culture positive cases, 48 cases yielded pure fungal isolates (Table 2). In 2 cases, there were mixed bacterial and fungal isolates, while 3 cases yielded two fungal isolates each. This study detected 56 fungal, and 12 bacterial isolates from 100 cases of corneal ulcers. The fungal isolates constituted $82.35 \%$ of total isolates. While the prevalence recorded from the southern, western and north-eastern India is between $21-37 \%$, it is only $7.3 \%$ from Chandigarh. In a larger study of 3528 microbial keratitis cases in Delhi (north India), the prevalence of fungal keratitis was reported to be $24.3 \%$ (Rautaraya et al., 2011).

Table.1 Direct smear positivity vs. culture positivity

\begin{tabular}{|l|l|l|l|l|l|}
\hline Direct Smear Positivity & No. & $\%$ & \multirow{2}{*}{ Fungal Culture Positivity } & No. & $\%$ \\
\cline { 2 - 3 } & 49 & $49 \%$ & & 56 & $56 \%$ \\
\hline
\end{tabular}

$49 \%$ of cases were positive in direct smear $(\mathrm{KOH})$, and $56 \%$ of cases gave growth in culture.

Table.2 Fungal pathogens isolated

\begin{tabular}{|l|l|l|}
\hline Species & No. of isolates & $\%$ \\
\hline Aspergillus spp & 22 & 39.29 \\
\hline Fusarium spp. & 9 & 16.07 \\
\hline Penicillium spp. & 4 & 7.14 \\
\hline Candida albicans & 2 & 3.57 \\
\hline Candida tropicalis & 1 & 1.79 \\
\hline Mucor spp. & 2 & 3.57 \\
\hline Acremonium & 3 & 5.36 \\
\hline Nigrospora & 2 & 3.57 \\
\hline Curvularia spp. & 2 & 3.57 \\
\hline Phoma spp. & 2 & 3.57 \\
\hline Cladosporium & 1 & 1.79 \\
\hline Dreschlera & 1 & 1.79 \\
\hline Lasiodiplodia theobromae & 1 & 1.79 \\
\hline Aureobasidium pullulans & 1 & 1.79 \\
\hline Fonsecaea spp. & 1 & 1.79 \\
\hline Unidentified Hyphae & 2 & 3.57 \\
\hline Total & 56 & \\
\hline
\end{tabular}

Among the 56 fungal isolates, 22 belonged to Aspergillus spp. Of this, Aspergillus flavus isolated (10 cases)was the most common isolate. Second most common was Fusarium spp.

Table.3 Aspergillus spp. isolated

\begin{tabular}{|l|l|l|}
\hline Aspergillus spp. & No. of isolates & $\%$ \\
\hline Aspergillus flavus & 10 & 45.45 \\
\hline Aspergillus fumigatus & 6 & 27.27 \\
\hline Aspergillus niger & 6 & 27.27 \\
\hline Total & 22 & $100 \%$ \\
\hline
\end{tabular}


Table.4 Corneal ulcers: other predisposing factors

\begin{tabular}{|l|l|l|}
\hline Sl. no. & Predisposing factors & No \\
\hline 1 & DM & 8 \\
\hline 2 & History of fall and keratitis in the past & 4 \\
\hline 3 & Facial Palsy & 2 \\
\hline 4 & Dry eye & 1 \\
\hline 5 & Lid abnormality & 1 \\
\hline Total & 16 \\
\hline
\end{tabular}

Table.5 Ocular trauma: causes of injury

\begin{tabular}{|l|l|l|}
\hline Sl. No. & Traumatic Agents & No \\
\hline 1 & Particulate material (Sand, Dust) & 29 \\
\hline 2 & Wooden Stick & 9 \\
\hline 3 & Building Material & 10 \\
\hline 4 & Insects & 1 \\
\hline 5 & Fingernails & 1 \\
\hline 6 & Dirty Water & 1 \\
\hline 7 & Vegetative matter & 5 \\
\hline Total & & 56 \\
\hline
\end{tabular}

Table.6 Corneal ulcers: seasonal variation

\begin{tabular}{|l|l|l|}
\hline Sl. No. & Months & Fungal isolation \\
\hline 1 & Dec, Jan, Feb & 12 \\
\hline 2 & Mar, Apr, May & 4 \\
\hline 3 & Jun, Jul, Aug & 32 \\
\hline 4 & Sep, Oct, Nov & 8 \\
\hline Total & 56 \\
\hline
\end{tabular}

The possible reason for reduced bacterial corneal ulcers might be more successful treatment of bacterial corneal ulcers in the peripheral centers and/or by general practitioners. Since this study is conducted at tertiary referral eye care center, the large number of fungal ulcers reported may be due to their prolonged course and poor response to available topical medications. $49 \%$ of the cases included in the study were direct smear - $10 \% \mathrm{KOH}$ positive, and $56 \%$ of cases yielded fungal isolates (Table 1). This shows $\mathrm{KOH}$ wet mount is a simple and sensitive method for diagnosis. Consequently, about 7 cases were missed on microscopic examination, which were positive in culture for fungi. But, 3 cases gave a positive microscopy for fungi without yielding fungal growth in culture. These would have been either artifacts, or these fungi must have been suppressed due to administration of topical antifungals. Study done at L.V. Prasad Eye Institute concludes Gram staining is much inferior to $10 \% \mathrm{KOH}$ mounts for visualization of fungal hyphae (Srinivasan et al., 1997).

Among the 56 fungal isolates, Aspergillus spp. was the commonest. Aspergillus species constituted $39.28 \%$ of the total fungal isolates. Aspergillus was speciated based on their macroscopic and microscopic morphological features. Accordingly $17.85 \%$ 
was Aspergillus flavus, and $10.71 \%$ Aspergillus fumigatus and Aspergillus niger respectively. In 1993, Chander et al., (1993) (Chandigarh) reported $35 \%$ of fungal isolates from corneal ulcers to be Aspergillus. In other studies Panda et al., (1997) from New Delhi (1997), Dunlop et al., (1994) from Bangladesh, Vemuganti et al., from Hyderabad have all reported Aspergillus as the commonest isolate in corneal ulcer.

Fusarium was the second commonest isolate, next to Aspergillus in this study. Similar findings were obtained in studies done in South India by Gar et al., from the Prasad Eye Institute, Hyderabad (37.6\%), and from Iran (Badiee, 2013; Thomas et al., 2013; Zubair et al., 2013).

Four corneal ulcers yielded Pencillium spp. in this study. This is $7.14 \%$ of the total fungal isolates. This is supported by Panda et al., who reported $7 \%$ of fungal isolates to be Pencillium spp. in his study at New Delhi (Chander et al., 1993).

Curvularia spp. is the commonest dematiaceous fungi in most of the studies conducted worldwide and in India. However in this study, there were 2 Curvularia isolates. This fungus is known to occur in the hot, humid, summer months Wilhelmus et al., (Zubair et al., 2013).

In a study in North India, Curvularia was the causative organism in $30.43 \%$ of cases followed by Fusarium (21.73\%) (Sanjeevani et al., 2010).

Acremonium spp. is one of the commonest hyalohyphomycete causing keratitis. Laspina et al., (Paraguay) reported this organism as the commonest fungal isolate from corneal ulcers. Another study from Madras Venugopal et al., (Sharma et al., 2008) reports Acremonium as the second commonest fungal isolate. Acremomium spp is also isolated in post-traumatic keratitis cases in Kancheepuram district of Tamil Nadu. In this study, there were 3 isolates of Acremonium spp $(5.36 \%)$. In this study, Candida spp. constituted $5.36 \%$ of the total fungal isolates. Candida was further speciated into Candida albicans, and Candida tropicalis. Candida albicans was the predominant isolate in a study conducted in Hyderabad (Narsani et al., 2012).

The other fungal isolates encountered in this study (Mucor spp., Nigrospora, Phoma spp. Cladosporium, Dreschlera, Lasiodiplodia theobromae, Aureobasidium pullulans, Fonsecaea spp.) though rare, are associated with corneal ulcers (Thomas et al., 2013).

Though majority of cases healed well following aggressive treatment, few cases ended up in corneal opacification, vascularization and perforation.

The present study was conducted in Regional Institute of Ophthalmology, Thiruvananthapuram. This study was an attempt to reveal the fungal in suppurative keratitis. Hundred patients - both OP and IP patients, with signs and symptoms of suppurative keratitis were studied from March 2012 to February 2013.

The following conclusions were derived from the present study:-

The male to female ratio was 2.125:1, indicating male preponderance.

The majority of patients were in the age group 41 to 50 years.

Manual laborers, followed by housewives and students were the dominant group in our study. 
The highest prevalence of suppurative keratitis was found in people living in rural areas.

Majority of patients belonged to low socioeconomic background.

Majority of patients presented in monsoon months.

$63 \%$ of patients included in this study had taken treatment before attending RIO. Majority had taken topical antibiotics alone.

$57 \%$ of patients included in this study gave history of ocular trauma, and particulate material like sand and dust were the most common traumatic agents. Diabetes Mellitus was another important predisposing factor.

Out of 100 cases, 56 were culture positive for fungus

Direct smear positivity was found in $49 \%$ of patients, whereas culture positivity of fungus was found in $56 \%$ of patients.

Aspergillus spp. was the most common fungal isolate followed by Fusarium spp., and Penicillium spp.

Although corneal ulceration is a very rare cause of mortality, it is one of the important causes of morbidity and disability in South Kerala, especially among lower socioeconomic groups. The present study gives an insight about the fungal and bacterial etiological agents of suppurative keratitis in this part of Kerala. The results of this study are similar to that in other parts of India. Few cases, especially Fusarium keratitis perforated within 1 to 2 weeks. So, early diagnosis and treatment with proper antifungal is of utmost importance. Microbiological culture, or a direct microscopy (KOH mount, or even a gram smear) is strongly advisable in this scenario. A complete microbiological investigation is mandatory and sight-saving in such a situation.

\section{References}

Anuradha, Padmaja. 2013. Bacterial Keratitis In and Around Visakhapatnam. Nat. J. Integrated Res. Med., 4(4): p. 38-41.

Arlappa, N. Epidemiological Overview of Preventable Blindness in India. National Institute of Nutrition, Indian Council of Medical Research.

Badiee, P. 2013. Mycotic Keratitis, a State-ofthe-Art Review. Jundishapur J. Microbiol., 6(5).

Bharathi, M.J., Ramakrishnan, R., Vasu, S., Meenakshi, R., Palaniappan, R. Epidemiological characteristics and laboratory diagnosis of fungal keratitis.

A three-year study. Indian $j$. Ophthalmol., 51(4): p. 315-21.

Biradar, S., Chandrashekhar, D.K., Gangane, R., Chandrakanth, C., Biradar, K.G., VinodKumar, C.S. 2012. Spectrum of microbial keratitis and antimicrobial susceptibility at tertiary care teaching hospital in north Karnataka. Int. J. Pharmaceutical and Biomed. Res., 3(2): p. 117-120.

Bozorgmehr, P., Anat, G., Darlene, M., Eduardo, A.C. 2011. Fungal Keratitis: One of Ophthalmology's Challenges: Epidemiology. Expert Review of Ophthalmol., 6(5): p. 529-540.

Butler, T.K., Spencer, N.A., Chan, C.C., Singh, G.J., McClellan, K. 2005. Infective keratitis in older patients: a 4 year review, 1998-2002. British J. Ophthalmol., 89(5): p. 591-6.

Chander, J., Chakrabarti, A., Sharma, A., Saini, J.S., Panigarhi, D.1993. Evaluation of Calcofluor staining in the diagnosis of fungal corneal ulcer. Mycoses, 36(7-8): p. 243-

Chander, J., Chakrabarti, A., Sharma, A., Saini, J.S., Panigarhi, D. 1993. Evaluation of Calcofluor staining in the diagnosis of fungal corneal ulcer. Mycoses, 36(7-8): p. 243-5. 
Dr.Srinivasan, E.V. 1969. Oration- Primary keratomycosis. Indian J. Ophthalmol., 17(5): p. 171-200.

Dunlop, A.A., Wright, E.D., Howlader, S.A., Nazrul, I., Husain, R., McClellan, K., et al. Suppurative corneal ulceration in Bangladesh. A study of 142 cases examining the microbiological diagnosis, clinical and epidemiological features of bacterial and fungal keratitis. Australian and New Zealand J. ophthalmol., 22(2): p. 105

Gebauer, A., McGhee, C.N., Crawford, G.J. 1996. Severe microbial keratitis in temperate and tropical Western Australia, Eye (London, England), 10(5): p. 575-80.

Hoffart, L., Dornadin, A., Drancourt, M. 2012. Epidemiology of microbial keratitis: A review of 508 cases. Acta Ophthalmologica, 90(s249).

Ibrahim, M.M., Vanini, R., Ibrahim, Martins, W.P., Carvalho, T.C., Silvestre de Castro, et al. 2011. Epidemiology and medical prediction of microbial keratitis in southeast Brazil. Arquivos Brasileiros de Oftalmologia, 74(1): p. 7-12.

Karsten, E., Watson, S., Foster, L.J.R. Diversity of Microbial Species Implicated in Keratitis: A Review. The Open Ophthalmol. J., 6: p. 110-124.

Kindo, A.J., Suresh, K., Premamalini, Anita, S., Kalyani, J. 2009. Fungus as an etiology in keratitis- our experience in SRMC. Sri Ramachandra J. Med., Jun; 2(2).

Kumari, N., Xess, A., Shahi, S.K. 2002. A study of keratomycosis: our experience. Indian J. Pathol. Microbiol., 45(3): p. 299-302.

Narayan, M. 2013. Prevalence of fungal keratitis in rural population - an Indian based, prospective; retrospective study. Int. J. General, Med. Pharmacy, 2(4): p. $37-46$.
Narsani, A.K., Nangdev, P.R., Surhio, S.A., Kumar, M., Jatoi, S.M. 2012. Demographic Pattern, Risk Factors, Clinical and Microbiological Characteristics of Fungal Keratitis. J. Liaquat Univ. Med. Health Sci., (JLUMHS), 11(01).

Nhung, P.H., Thu, A.T., Ngoc, L.H., Ohkusu, K., Ezaki, T. Epidemiology of Fungal Keratitis in North Vietnam. J. Clin. Experimental Ophthalmol., 3(7).

Panda, A., Sharma, N., Das, G., Kumar, N., Satpathy, G. 1997. Mycotic keratitis in children: epidemiologic and microbiologic evaluation. Cornea, 16(3): p. 295-9.

Punia, R.S., Kundu, R., Chander, J., Arya, S.K., Handa, U., Mohan, H. 2014. Spectrum of fungal keratitis: clinicopathologic study of 44 cases. Int. J. Ophthalmol., 7(1): p. 114-7.

Rautaraya, B., Sharma, S., Kar, S., Das, S., Sahu, S.K. 2011. Diagnosis and treatment outcome of mycotic keratitis at a tertiary eye care center in eastern India. BMC Ophthalmol.

Rautaraya, B., Sharma, S., Kar, S., Das, S., Sahu, S.K. 2011. Diagnosis and treatment outcome of mycotic keratitis at a tertiary eye care center in eastern India. BMC Ophthalmol.

Saha, R., Das, S. 2006. Mycological profile of infectious keratitis from Delhi. The Indian J. Med. Res., 123(2): p. 159-64.

Sanjeevani, R., Sengupta, S., Reddy, P.R., Ravindran, R.D., Krishnan, T.K. 2010. Incidence and Outcomes of Dematiaceous Vs Filamentous Fungal Keratitis - A Retrospective Analysis. In All India Ophthalmological Conference (AIOC), p. 218-222.

Schaefer, F., Bruttin, O., Zografos, L., GuexCrosier, Y. 2001. Bacterial keratitis: a prospective clinical and microbiological study. British J. Ophthalmol., 85(7): p. 842-847. 
Sharma, B., Gupta, R., Som, V., Kubrey, S.S., Anand, R., Kumar, K. 2013. Clinical profile of microbial keratitis causes and contributing factors. J. Evol. Med. Den. Sci., 2(51): p. 9939-9947.

Sharma, V., Purohit, M., Vaidya, S. 2008. Epidemiological study of Mycotic Keratitis. The Internet J. Ophthalmol. Visual Sci., 6(2).

Srinivasan, M., Gonzales, C.A., George, C., Cevallos, V., Mascarenhas, J.M., Asokan, B., et al., Epidemiology and etiological diagnosis of corneal ulceration in Madurai, South India. British J. Ophthalmol., 81: p. 965-

Tabatabaee, A., Mohajernezhadfard, Z., Daneshgar, F., Mansouri, M. 2013. Keratomycosis after incidental spillage of vegetative material into the eye: Report of two cases. Oman $J$. Ophthalmol., 6(2): p. 122-6.
Thomas, P.A., Kaliamurthy, J. 2013. Mycotic keratitis: epidemiology, diagnosis and management. Clinical microbiology and infection: the official publication of the European Society of Clin. Microbiol. Infect. Dis., 19(3): p. 210-20.

Thomas, P.A., Kaliamurthy, J. 2013. Mycotic keratitis: epidemiology, diagnosis and management. Clinical microbiology and infection: the official publication of the European Society of Clin. Microbiol. Infect. Dis., 19(3): p. 210-20.

Venugopal, P.L., Venugopal, T.L., Gomathi, A., Ramakrishna, E.S., Ilavarasi, S. 1989. Mycotic keratitis in Madras. Indian J. Pathol. Microbiol., 32(3): p. 190-7.

Zubair, A., Darlene, M., Anat, G. 2013. Current Thoughts in Fungal Keratitis: Diagnosis and Treatment. Curr. Fungal Infection Reports, 7(3): p. 209-218.

\section{How to cite this article:}

Anna Cherian, Honey Susan Paul, R. Jyothi and Ramani Bai, J.T. 2017. A study on Fungal Etiological Agents in Suppurative Keratitis. Int.J.Curr.Microbiol.App.Sci. 6(6): 1740-1749. doi: https://doi.org/10.20546/ijcmas.2017.606.202 